asthma, four studies demonstrated no relationship. Evidence from one uncontrolled study suggested symptoms of severe asthma may improve following combined surgical and medical management. Another uncontrolled study showed that allergic rhinitis symptoms improved following treatment of severe asthma with omalizumab.

Conclusions Though a unified airways model supports the relationship between rhinitis and asthma, evidence regarding severe asthma specifically is of low quality and Results are varied. Although it is likely that rhinitis symptoms improve alongside successful treatment of severe asthma, adequately powered randomised studies are necessary to substantiate this relationship.

\section{P230 DOES THE GLOBAL ASTHMA VISUAL ANALOGUE SCALE RELATE TO THE ASTHMA CONTROL QUESTIONNAIRE?}

S Jabbal, B Lipworth. University of Dundee, Dundee, UK

\subsection{6/thoraxjnl-2017-210983.372}

Control based asthma management results in improved asthma outcomes. The Asthma Control Questionnaire (ACQ-6), is a widely used and well validated metric which strongly predicts future exacerbations. ${ }^{1}$ It demarcates between controlled (C), partially controlled (P), and uncontrolled (U), based on cut point scores of $<0.75,0.75<1.5$, and $\geq 1.5$ respectively. The global asthma visual analogue scale (VAS) is a $10 \mathrm{~cm}$ continuum indicating the overall symptom burden. ${ }^{2}$ It discriminates GINA categories of $\mathrm{C}, \mathrm{P}$ and $\mathrm{U}$ as $<1.5,1.5<7.19$, and $\geq 7.19$ respectively. We evaluated how VAS relates to ACQ in terms of predefined GINA cut points. We analysed $\mathrm{n}=87$ patients who attended for asthma screening into clinical trials. $90 \%$ of patients were receiving ICS(mean BDP equiv $675 \mu \mathrm{g} /$ day), of whom $80 \%$ received ICS/LABA, 42\% with LTRA, mean FeNO 45 ppb, mean FEV1 89\%, and mean number +ve skin prick tests were 2. Overall Spearman's correlation was $0.62, \mathrm{p}<0.001$. Mean VAS levels for ACQ were: C: $2.2 \mathrm{~cm}$ (95\% CI 1.35-3.06), P: $2.56 \mathrm{~cm}$ (95\% CI 2.61-4.50), U: $5.27 \mathrm{~cm}$ (95\% CI 4.46-6.08), i.e., $<7.19 \mathrm{~cm}$ GINA defined cut off (figure 1). There was no significant difference between patients with $\mathrm{ACQ} \geq 1.5$ vs $<1.5$ for $\mathrm{FeNO}$ (51 ppb vs 41 $\mathrm{ppb}$ ), or BDP equiv dose $(674 \mu \mathrm{g}$ vs $543 \mu \mathrm{g})$. Chi-square test demonstrated a weak relationship between ACQ $\geq 1.5$ and GINA defined VAS cut off $\geq 7.19 \mathrm{~cm}$. A VAS $\geq 7.19$ had a sensitivity of $29 \%$ and specificity of $92 \%$ for detecting an $A C Q \geq 1.5$. ROC analysis, using ACQ to compare C vs U/P revealed an optimal cut point for VAS of 1.95 (AUC 0.8 , sensitivity $88 \%$, specificity $68 \%$ ). Comparing $\mathrm{U}$ vs $\mathrm{P} / \mathrm{C}$ revealed VAS cut point of $3.2 \mathrm{~cm}$ (AUC 0.7 , sensitivity 71\%, specificity $57 \%)$. We conclude that the GINA defined VAS cut off $(\geq 7.19)$ is a poor predictor of control in relation to an $A C Q \geq 1.5$. Hence, further evaluation is required to define the VAS threshold in relation to control defined by ACQ rather than GINA.

Figure Legend Distribution of ACQ control categories relative to VAS levels.

\section{REFERENCES}

1. Meltzer et al. JACI 2011;127:167-172.

2. Ohta et al. J Asthma 2013;50:514-521.

\section{Closing the flood gates of the pleura}

\section{P231 THE USE OF INDWELLING PLEURAL CATHETERS IN PATIENTS WITH MALIGNANT PLEURAL EFFUSION AND UNEXPANDABLE LUNG}

P Halford, R Bhatnagar, NA Maskell. University of Bristol, Bristol, UK

\subsection{6/thoraxjnl-2017-210983.373}

Introduction British Thoracic Society (BTS) guidelines suggest that Indwelling Pleural Catheters (IPCs) are the best treatment for malignant pleural effusion (MPE) associated with unexpandable lung (UL), where pleurodesis is contraindicated. Although, comprehensive data highlight the benefit of IPCs in MPE, their efficacy in patients with UL is less well known.

Method We conducted a systematic review following searches of the MEDLINE, EMBASE and Web of Science databases up until June 2017. Studies specifically reporting IPC use in patients with MPE and UL were identified.

Results 24 studies using IPCs in MPE in general were analysed, 15 of which stated the proportion with UL. Only 3 trials specifically reported outcomes in patients with UL, ranging from $11-52$ patients. $77 \%-94 \%$ of UL patients had symptomatic benefit with IPC. Complication rate ranged from 15\%$56 \%$ depending on criteria used, common complications reported included pain, cellulitis, catheter leak and occlusion. Self-pleurodesis in the largest trial occurred in $42.3 \%$ Mean hospital stay was three days, however only one study reported this. Mortality was mentioned in two papers, with a mean survival of 126 days post-IPC insertion. Incidence of UL in the MPE population undergoing IPC was calculable in five studies, and ranged from $7.5 \%-41 \%$. Differences were evident depending on criteria for diagnosing UL. One study using video-assisted thoracoscopy in 127 patients had an incidence of $41 \%$ whereas another study using post-procedure radiography found UL in 40/295 IPCs (13.6\%).

Conclusion There appears to be symptomatic benefit in using IPCs in UL patients. Although complications tended to be relatively minor, their rates appear increased compared to the broader IPC population. However, the lack of consensus in diagnostic criteria for UL, reflected by the varied incidence reported, makes application harder to interpret. Further evidence is needed to establish a consistent approach to UL diagnosis, along with more robust studies addressing incidence. The use of IPCs in this population also needs further, prospective, study, with a focus on patient-centred outcomes and device efficacy. This would ideally include further validation of the finding that IPC's in UL can lead to pleurodesis in a large number of patients.

\section{P232 FACTORS PREDICTING OUTCOMES OF TALC PLEURODESIS IN THOSE WITH MALIGNANT PLEURAL EFFUSIONS AT A BELFAST TEACHING HOSPITAL}

E Keelan, R Whitaker, N Magee. Regional Respiratory Centre, Belfast City Hospital, Lisburn Road, Belfast, Ireland

\subsection{6/thoraxjnl-2017-210983.374}

Introduction Malignant pleural effusions (MPE) are a frequent consequence of advanced malignancy, associated with poor prognosis. The British Thoracic Society endorse intercostal drainage and talc pleurodesis as first line management of MPE 